\title{
Mechanical modulation of single-electron tunneling through molecular-assembled metallic nanoparticles
}

\author{
Yongqiang Xue * and Mark A. Ratner \\ Department of Chemistry and Materials Research Center, \\ Northwestern University, Evanston, Illinois 60208, USA
}

(Dated: February 8, 2020)

\begin{abstract}
We present a microscopic study of single-electron tunneling in nanomechanical double-barrier tunneling junctions formed using a vibrating scanning nanoprobe and a metallic nanoparticle connected to a metallic substrate through a molecular bridge. We analyze the motion of single electrons on and off the nanoparticle through the tunneling current, the displacement current and the charging-induced electrostatic force on the vibrating nanoprobe. We demonstrate the mechanical single-electron turnstile effect by applying the theory to a gold nanoparticle connected to the gold substrate through alkane dithiol molecular bridge and probed by a vibrating platinum tip.

PACS numbers: 85.35.Gv,85.85.+j,85.65.+h,73.63.-b
\end{abstract}

Introduction.- Nanoelectromechanical devices that combine mechanics with electronics are of great interests for applications in electronics, precision measurement and sensors $\frac{1}{\underline{1}}$ Among the experimental implementations, mechanical single-electron devices that explore the interplay between the macroscopic motion of a nanomechanical element and the quantized single-electron transfer have attracted much attention for revealing new mechanisms of electron transport and schemes of mechanical detection at the quantum-limit. $\stackrel{2}{2}$ In particular, the shuttle mechanism of quantized charge transfer has been proposed theoretically ${ }^{3.4}$ and studied experimentally $y^{\frac{5}{5}}$ utilizing a variety of nanomechanical elements.

The model system considered in the original proposal ${ }^{3}$ for a mechanical single-electron shuttle consists of a small metal cluster connected to the electrodes through mechanically soft organic linkers. A periodic self-oscillation of the cluster in conjunction with the cluster charging/decharging is predicted for sufficiently large bias voltages, leading to an average current proportional to the self-oscillation frequency $\underline{\underline{3}}$ Two factors neglected in the original proposal may complicate the analysis and prevent the observation of the shuttle effect in molecularassembled single-electron devices: ${ }^{6}$ (1) At small oscillation amplitude, the cluster displacement modulates the metal-molecule bond length/strength rather than the molecule core. The exponential dependence of the tunneling resistance on the displacement of the metal island may not hold, ${ }^{7}$ (2) At large oscillation amplitude, the internal structure of the linker molecule may be distorted due to the forces induced by the change in metal-molecule bond, the net charges on the cluster and the applied electrical field. The shuttle mechanism of electron transfer has also been used to interpret recent experiment on a $C_{60}$ single-electron transistor, $\stackrel{8.9}{~ b u t ~ a l t e r n a t i v e ~ e x p l a n a-~}$ tions ${ }^{10}$ also exist. Recently it has been suggested that measurement of shot noise spectrum 11 and full counting statistics 12 may help in elucidating the mechanism involved.

A simpler way of incorporating mechanical degree of freedom into single-electron devices is to couple one of the tunnel junctions to a nanomechanical oscillator, ${ }^{13}$ using e.g., the microcantilever tip of a conducting Atomic Force Microscope (AFM) or hybrid Scanning Tunneling Microscope (STM)/AFM ${ }^{14.15 .16}$ The model system is illustrated schematically in Fig. 1(a), where the nanoparticle is connected to the substrate through an organic molecule (assumed rigid), while tunneling across the top contact can be modulated mechanically through the vibrating tip. The introduction of a vibrating tunnel contact leads to tunneling and displacement currents flowing simultaneously in accordance with the probe vibration, 17.18 which can be measured separately using a twophase lock-in amplifier ${ }^{14}$ The discrete-electron tunneling also induces an electrostatic force on the tip 14.15.16,19 Since both the tunneling/displacement currents and the force on the microcantilever tip can be measured as a function of the bias voltage, useful information regarding the interplay among tip vibration, discrete electron motion and metal-molecule interaction can be extracted, which may potentially allow applications in displacement detection and chemical/bio-sensing. The purpose of this paper is thus to present a theoretical analysis of such nanomechanical double-barrier tunneling junctions using realistic atomic-scale models.

Master-equation approach to single-electron tunneling through molecular-assembled metallic nanoparticles.We consider periodic vibration of the tip, with the tipnanoparticle distance being $x(t)=d_{0}+d_{1} \cos 2 \pi f t$. The coupled molecule-nanoparticle-vibrating tip system is described by the following Hamiltonian:

$$
H=H_{S}+H_{T}+H_{I}+V_{e s}+H_{m o l}+H_{T_{S}}+H_{T_{T}},
$$

where $H_{\alpha}=\sum_{k} \epsilon_{k \alpha} a_{k \alpha}^{\dagger} a_{k \alpha}(\alpha=S, T)$ and $H_{I}=$ $\sum_{l} \epsilon_{l I} c_{l I}^{\dagger} c_{l I}$ describe the noninteracting electrons in the substrate (S), tip (T) electrodes and on the central island (I) respectively. We model both the electrodes and the nanoparticle as infinite electron reservoirs with electrochemical potential $\mu_{\alpha}, \alpha=S, T, I$. The indices $k$ and $l$ enumerate the electron states of the electrodes and the nanoparticle. We describe the linker molecule using an ef- 

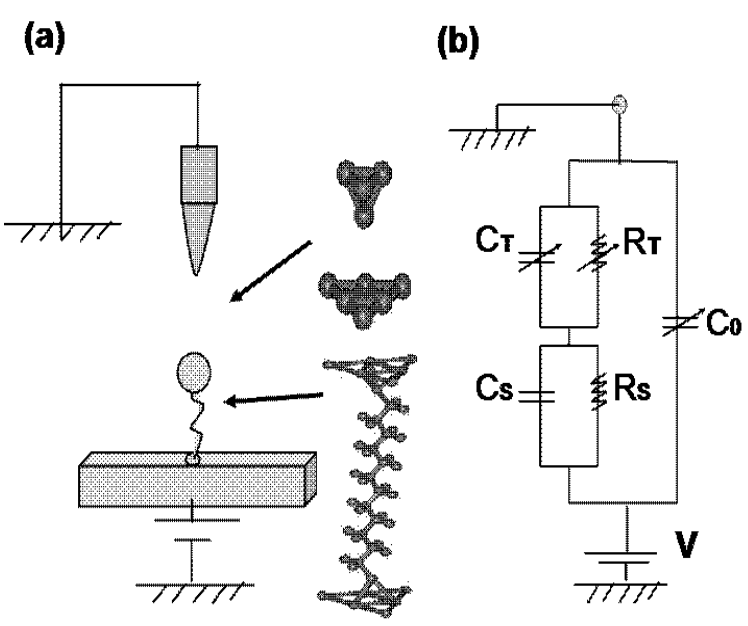

FIG. 1: (Color online) (a) shows schematic illustration of the nanomechanical double-barrier tunnel junctions formed using a vibrating platinum tip and a gold metallic nanoparticle connected to the gold substrate through an alkane dithiol molecular bridge with 12 alkane units (AK12). The platinum tip is modeled as a 4-atom pyramid sitting on top of the semi-infinite substrate. (b) shows the equivalent circuit model of the system. Note that the substrate junction capacitance $\left(C_{S}\right)$ and resistance $\left(R_{S}\right)$ are fixed, while the tip junction capacitance $\left(C_{T}\right)$ and resistance $\left(R_{T}\right)$ are time-dependent due to the tip vibration. The tip-substrate capacitance $\left(C_{0}\right)$ is also time-dependent.

fective single-particle Hamiltonian $H_{m o l}=\sum_{i} \epsilon_{i} b_{i m}^{\dagger} b_{i m}$. The electrostatic part of the energy is $V_{e s}\left(q_{S}, q_{T}\right)=$ $\frac{q^{2}}{2 C_{\Sigma}}+q_{S} V_{S}+q_{T} V_{T}, C_{\Sigma}=C_{S}+C_{T}$, where $C_{S(T)}$ and $q_{S(T)}$ are the capacitance and charge of the substrate (tip) junction respectively ${ }^{20}$ The voltage drop across the substrate (tip) junction is determined from the tip-substrate bias voltage $V$ by $V_{S(T)}=\frac{C_{T(S)}}{C_{\Sigma}} V$. The net charge on the central island is $q=q_{S}-q_{T}=-e\left(n+n_{x}\right)$, where $e n_{x}$ is the background charge and $e n$ is the quantized island charge. Unlike the conventional single-electron devices where the background charge is typically induced by charged impurities embedded in the insulating layer, here an intrinsic background charge $n_{x}$ can be induced by the charge transfer between the molecule and the central island during the formation of the substrate junction, ${ }^{21}$ and can be obtained from microscopic molecular junction calculations 22

The transfer of single-electrons is mediated by tunneling through the molecular (substrate) junction $H_{T_{S}}=$ $\sum_{i k}\left(t_{i m ; k S} b_{i m}^{\dagger} a_{k S}+t_{i m ; l I} b_{i m}^{\dagger} c_{l I} e^{-i \phi_{S}}+\right.$ H.C. $)$, and the tip junction $H_{T_{T}}(t)=\sum_{l k}\left(t_{l I ; k T}(x(t)) a_{k T}^{\dagger} c_{l I} e^{-i \phi_{T}}+H . C.\right)$, where we have shown explicitly the time-dependence of the tip-nanoparticle coupling through the tip position $x(t)$. The phase operator $\phi_{S(T)}$, canonically conjugate to the charge $q_{S(T)}$ on the substrate (tip) junction, keeps track of the quantized electron tunneling since $\left[\phi_{S(T)}, q_{S(T}\right]=i e$ and $e^{i \phi_{S(T)}} q_{S(T)} e^{-i \phi_{S(T)}}=q_{S(T)}-$

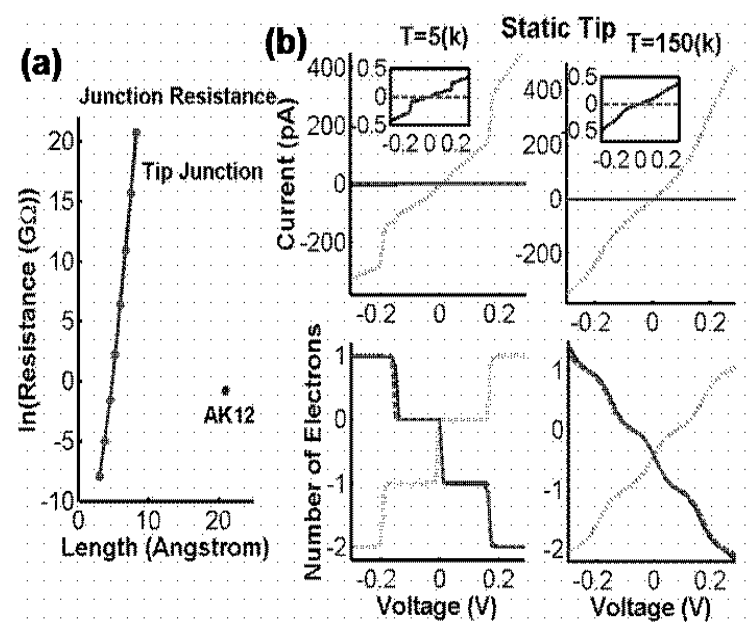

FIG. 2: (a) shows the molecular junction and tip junction resistance as a function of the junction length. The tip junction resistance increases exponentially with tip-nanoparticle distance as the tip remains weakly coupled to the nanoparticle during the tip vibration cycle. (b) shows the static currentvoltage $(\mathrm{I}-\mathrm{V})$ and excess island charge-voltage (n-V) characteristics of the double-barrier tunnel junctions for a $10(\mathrm{~nm})$ diameter nanoparticle at tip-nanoparticle distance of $3.0 \AA$ (dotted line), 6.0 (solid line) and $9.0 \AA$ (dashed line) for temperatures of $T=5,150 \mathrm{~K}$ respectively. The inset shows the magnified view of $\mathrm{I}-\mathrm{V}$ characteristics at $6.0 \AA$ and $9.0 \AA$. Note that at $x=6.0 \AA$, we already have $R_{T}>>R_{S}$, so the $\mathrm{n}-\mathrm{V}$ characteristics remains approximately the same as $x$ increases further.
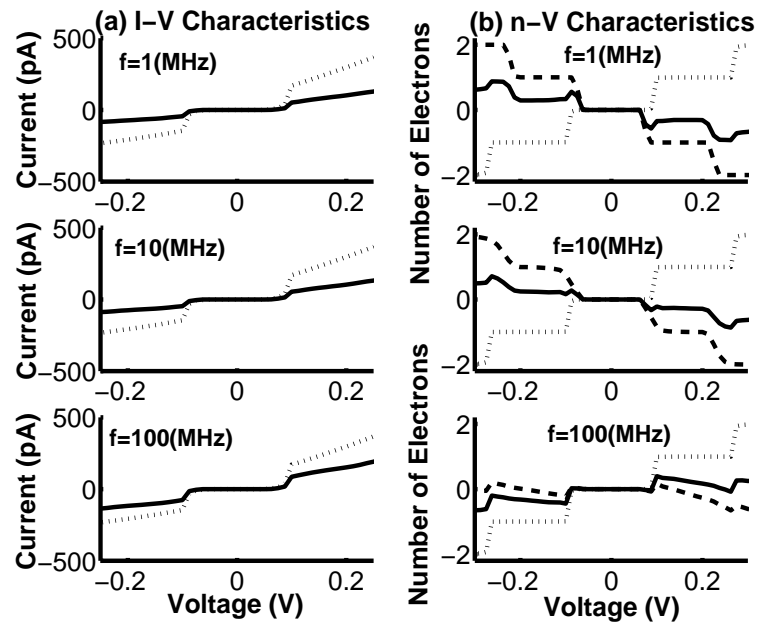

FIG. 3: (a) shows the time-averaged tunneling I-V characteristics (solid line) and the instantaneous tunneling I-V characteristics at $x=3.0 \AA$ (dotted line) at three tip vibrating frequencies of $f=1,10,100 \mathrm{MHz}$. The tunnel currents are virtually independent of the tip vibrating frequency. (b) shows the time-averaged $\mathrm{n}-\mathrm{V}$ characteristics (solid line), the instantaneous $\mathrm{n}-\mathrm{V}$ characteristics at $x=3.0 \AA$ (dotted line) and $x=9.0 \AA$ (dashed line) respectively. The instantaneous values are the values obtained at the moments when the tip moves to $x=3.0 \AA$ or $9.0 \AA$ 


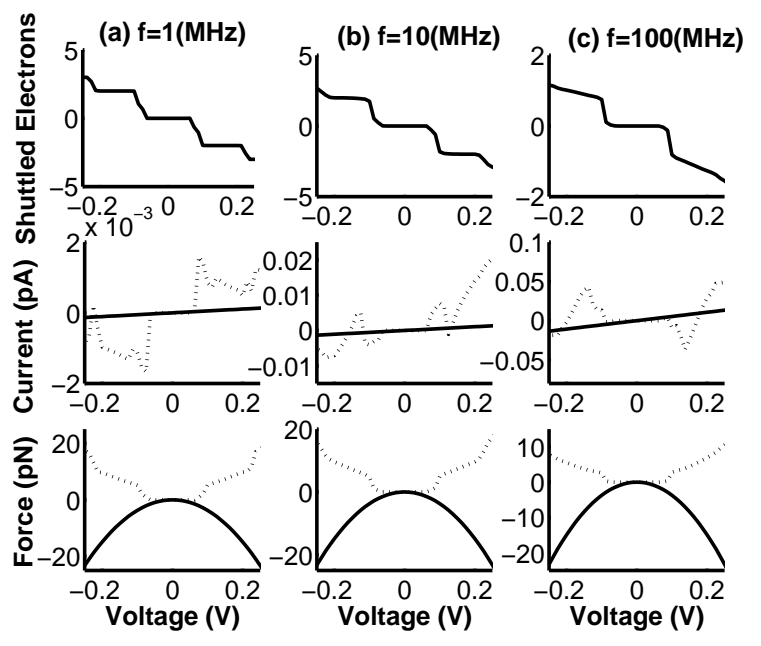

FIG. 4: Upper figures show the average number of shuttled electron per cycle of tip vibration, middle figures show the average displacement current, and lower figures show the average electrostatic force on the metallic tip as a function of tip-substrate bias voltage at tip vibrating frequencies of $f=1,10,100(M H z)$. In the middle figures, we have shown both the capacitive part (solid line) and "Coulomb Blockade" part (dotted line) of the displacement current. The capacitive part increases linearly with voltage and tip vibrating frequency. But the "Coulomb Blockade" part oscillates with increasing voltage. In the lower figures, we have shown both the capacitive part $F_{C a p}$ (solid line) and discrete-electron part $F_{C B}$ (dotted line) of the electrostatic force on the tip. Note that the step-wise increase in the shuttled electrons in the upper figures correlates with the step-like increase in discreteelectron force $F_{C B}$ in the lower figures.

$e e^{23.24}$ The electrostatic energy change after tunneling of an electron through the substrate(tip) junction is thus $\Delta_{n}=e^{i \phi_{S(T)}} V_{e s} e^{-i \phi_{S(T)}}-V_{e s}=e V_{S(T)}+\frac{(q-e)^{2}}{2 C_{\Sigma}}-$ $\frac{q^{2}}{2 C_{\Sigma}} \cdot 20.23$

We describe single-electron transport using the master equation: 4.18 .25

$$
\begin{aligned}
\frac{d P_{m}(t)}{d t} & =P_{m+1}(t) \Gamma^{-}\left(\Delta_{m}, t\right)+P_{m-1}(t) \Gamma^{+}\left(\Delta_{m-1}, t\right) \\
& -P_{m}(t)\left(\Gamma^{-}\left(\Delta_{m-1}, t\right)+\Gamma^{+}\left(\Delta_{m}, t\right)\right)
\end{aligned}
$$

where $P_{m}$ is the probability of finding $m$ excess electrons on the nanoparticle and $\Gamma^{+(-)}=\Gamma_{S}^{+(-)}+$ $\Gamma_{T}^{+(-)}$. The rate for transition $m \rightarrow m \pm 1$ due to electron tunneling through the substrate (tip) junction, $\Gamma_{S(T)}^{+(-)}$, is obtained from the electron transmission coefficient of the corresponding junctions us$\operatorname{ing} \Gamma_{S(T)}^{+}(w, t)=\frac{2}{h} \int d E T_{S(T)}(E, V ; t) f_{S(T)}(E)(1-$ $\left.f_{I}(E-w)\right)$ and $\Gamma_{S(T)}^{-}(w, t)=\frac{2}{h} \int d E T_{S(T)}(E, V ; t)(1-$ $\left.f_{S(T)}(E)\right) f_{I}(E-w),{ }^{21}$ where $w=\Delta_{m}$ or $\Delta_{m-1}$. The transmission coefficient $T_{S(T)}$ can be determined from the standard Non-Equilibrium Green's Function theory ${ }^{26}$ as developed for molecular tunnel junctions. ${ }^{21,22}$ Here the time dependence in the tunneling rate $\Gamma^{+(-)}$is introduced through the time-dependent tunneling rate across the tip junction $\Gamma_{T}^{+(-)}$due to the tip vibration $x(t)$. Since the tunneling time for electron tunneling across the tip junction is much smaller than the tip vibration period, the time-dependence in $T_{T}$ indicates that we calculate the transmission coefficient using the tunneling Hamiltonian $H_{T_{T}}(t)$ at the instantaneous tip position $x(t)$.

The tunneling current across the tip junction and the average excess electron number on the nanoparticle at time $t$ are given by $I_{T}^{T \text { un }}(t)=e \sum_{m} P_{m}(t)\left[\Gamma_{T}^{+}\left(\Delta_{m}, t\right)-\right.$ $\left.\Gamma_{T}^{-}\left(\Delta_{m-1}, t\right)\right]$ and $n(t)=\sum_{m} m P_{m}(t)$ respectively. The displacement current flowing through the vibrating tip is due to time-variation of system charges and is fixed by electrostatic considerations 18,23 :

$$
\begin{aligned}
I_{T}^{\text {Dis }}(t) & =\dot{C}_{0} V+\frac{d q_{T}}{d t}=I_{C a p}(t)+I_{C B}(t) \\
& =\frac{d}{d t}\left(C_{0}+\frac{C_{S} C_{T}}{C_{S}+C_{T}}\right) V+e \frac{d}{d t}\left(\frac{C_{T}}{C_{S}+C_{T}}(n(t)+n(3))\right.
\end{aligned}
$$

where $C_{0}$ is the capacitance between the tip and the substrate. The first term $I_{C a p}$ is the displacement current induced by the direct capacitive coupling between the tip and the substrate and the series capacitance of the double-barrier junction. The second term $I_{C B}$ gives the "Coulomb blockade" component of displacement current due to the time variation of the excess electrons on the nanoparticle. The electrostatic force on the oscillating tip can also be divided into the capacitive force $F_{C a p}$ and the discrete-electron force $F_{C B}$ as follows:

$$
\begin{aligned}
F_{t i p} & =-\frac{1}{2} \frac{d C_{0}}{d x} V^{2}-\frac{1}{2} \frac{d\left(q_{T} V_{T}\right)}{d x}=F_{C a p}+F_{C B} \\
& =-\frac{1}{2} \frac{d}{d x}\left(C_{0}+C_{T}\left(\frac{C_{S}}{C_{\Sigma}}\right)^{2}\right) V^{2}-\frac{e}{2} \frac{d}{d x}\left(\frac{C_{S} C_{T}}{C_{\Sigma}^{2}}\left(n+n_{x}\right)\right)\left({ }^{2}\right)
\end{aligned}
$$

Since our main interests here are in electron transport and charging-induced forces, we neglect non-voltage dependent forces like the long-range van der Waals force. Since we consider transport within the Coulomb blockade regime, the tip remains weakly coupled to the nanoparticle during the entire cycle of vibration, and we neglect also the short-range contact force that may arise from the tip-nanoparticle bonding. Both type of forces would have been important if we want to study the detailed oscillation dynamics of the cantilever 16

Device model. - We apply the theory to single-electron tunneling through a $10(\mathrm{~nm})$-diameter gold nanoparticle connected to the gold $\langle 111\rangle$ substrate through an alkane dithiol molecule with 12 alkane units (AK12) and probed using a vibrating platinum tip. Electron transport through gold-dithiol molecule-gold junctions have been recently studied in detail using a first-principles selfconsistent matrix Green's function theory, ${ }^{22}$ from which we obtain the intrinsic background charge $e n_{x}$ induced by the charge transfer from the molecule to the nanoparticle and the transmission coefficient for electron tunneling through the metal-molecule-nanoparticle junction. 
To summarize, the gold electrodes are modeled as semiinfinite $\langle 111\rangle$ single-crystals. Six nearest-neighbor gold atoms on each metal surface (twelve gold atoms overall) are included into the "extended molecule" where the selfconsistent calculation is performed. The rest of the electrodes (with the six atoms on each surface removed) are considered as infinite electron reservoirs, whose effects are included as self-energy operators. The calculation is performed using the Becke-Perdew-Wang parameterization of density-functional theory ${ }^{27}$ and appropriate pseudopotentials with corresponding optimized Gaussian basis sets ${ }_{2}^{22}$ We find that the resistance of the AK12 molecular junction is $R_{S} \approx 300(M \Omega)$, and the background charge is $n_{x} \approx-0.45^{28}$

Since the tip geometry in STM/AFM is often not well characterized, we model the $\mathrm{Pt}$ tip as a 4-atom Pt pyramid sitting on top of the $\langle 111\rangle \mathrm{Pt}$ substrate. The tunneling matrix elements are obtained using the semiempirical Extended Huckel Theory (EHT) ${ }^{29}$ and considering coupling between the Pt pyramid and six neighbor gold atoms on the surface of the gold nanoparticle, with the apex $\mathrm{Pt}$ atom sitting in front of the center of the six gold atoms arranged following the $\langle 111\rangle$ gold surface geometry (Fig. 1(a)). The calculated tip junction resistance $R_{T}$ as a function of the tip apex-nanoparticle distance is shown in Fig. 2(a), which increases exponentially with the tip-nanoparticle distance once it falls within the weak-coupling regime. For capacitance modeling, such atomic-scale model is not needed. The tip is instead replaced by a $1 \mu m$-radius sphere, which takes into account approximately the tip curvature effect and is common in AFM research. ${ }^{16.30}$ Here both the substrate and tip junction capacitance are obtained from classical electrostatics of conducting sphere sitting in front of the conducting substrate,, 30 which gives the substrate junction capacitance of $0.9 a F$, the tip junction capacitance of $0.97 a F$ and tip-substrate capatitance of $0.34 \mathrm{fF}$ at tipnanoparticle distance $x=9.0 \AA$ respectively.

Results and their interpretation.- We assume the tip vibration being described by oscillation amplitude of $d_{1}=3.0 \AA$ and average tip-nanoparticle distance of $d_{0}=$ $6.0 \AA$. The device functions as a nanomechanical singleelectron turnstile, which can be understood by examining the static (tip not moving) excess island electronvoltage $(\mathrm{n}-\mathrm{V})$ characteristics at different tip-nanoparticle distances $x$ (Fig. 2 (b)). We find that the trend of the charging state on the nanoparticle is reversed as the tipnanoparticle $x$ increases from $3.0 \AA$ to $9.0 \AA$. This is because at $x=3.0 \AA$, the tip junction resistance is much smaller than the substrate junction resistance while at $x=9.0 \AA$, the tip junction resistance becomes much larger than the substrate junction resistance (Fig. 2](a)). As the tip starts vibrating at $x=d_{0}+d_{1}=9.0 \AA$, the nanoparticle will be charged by discrete-electron tunneling across the substrate junction if the bias voltage is large enough to overcome the charging energy. As the tip moves close to the nanoparticle at $x=d_{0}-d_{1}=3.0 \AA$, the tip junction becomes sufficiently conductive $\left(R_{T}<<R_{S}\right)$ allowing the stored excess charge on the nanoparticle to be transferred onto the tip. A discrete number of electrons is thus being shuttled across the system per tip vibrating cycle. Note that the $\mathrm{n}-\mathrm{V}$ characteristics at $x=d_{0}=6.0 \stackrel{\circ}{ }$ is similar to that at $x=9.0 \AA$ because $R_{T}$ is already much larger than $R_{S}$ at $x=6.0 \stackrel{\AA}{A}$, which is also the case during most part of the tip vibration cycle.

We solve the rate equation (Eq. 2) numerically, 31 from which we obtain the time-dependent tunneling current, the displacement current and the electrostatic force on the Pt tip. We calculate the probability $P_{\Delta}(m)$ that $m$ electrons have been transferred during one cycle of tip vibration from $P_{\Delta}(m)=\sum_{k} P_{i}(k) P\left(k-m \mid k, t_{0}+\right.$ $1 / f$ ) (f is the vibrating frequency),, 4 where $P_{i}(k)$ is the possibility of finding $\mathrm{k}$ electrons on the nanoparticle at time $t_{0} . P\left(k-m \mid k, t_{0}+1 / f\right)$ is the conditional probability of finding $k-m$ electrons on the nanoparticle after one cycle of tip oscillation if there are $k$ electrons at initial time $t_{0}$, which is obtained by solving the rate equation Eq. 2 with the initial condition $P\left(k, t_{0}\right)=1, P\left(i, t_{0}\right)=$ $0, i \neq k$. . The average number of electrons being shuttled across the system is $n_{\text {shuttle }}=\sum_{m} m P_{\Delta}(m)$.

The average (time-average over one cycle of the tip vibration) tunneling current-voltage (I-V) flowing across the tip junction and excess island electron-voltage (n-V) characteristics of the vibrating double-barrier junctions at temperature of $5 K$ are shown in Fig. 3 for three tip vibrating frequencies $f=1,10,100(\mathrm{MHz})$. For comparison, we have also shown the instantaneous tunneling I-V characteristics when the tip is closest to the nanoparticle $(x=3.0 \AA)$ in Fig. 3(a), the instantaneous n-V characteristics at two different tip-nanoparticle distances of $x=3.0$, and $9.0 \AA$ in Fig. 3 (b). The instantaneous values at $x=3.0 \AA 9.9 .0 \AA$ are the values obtained at the moment when the tip moves to $x=3.0 \AA$ ․ $9.0 \AA$ respectively. Since the instantaneous tunneling current of the vibrating double-barrier junction decreases rapidly as tip moves away from the nanoparticle, and the tip velocity approaches zero as tip moves closer to the nanoparticle, the average tunneling I-V characteristics is similar in both magnitude and voltage dependence to the instantaneous tunneling I-V characteristics at $x=3.0 \AA$ at all three tip frequencies. Note that the tunneling currents shown here are virtually independent of the tip vibrating frequency, since the time it takes for electron to tunnel across the tip junction is the smallest of all time scales involved here and can be considered as instantaneous compared to the tip vibration.

The $n-V$ characteristics show stronger tip-frequency dependence than the tunneling I-V characteristics. The instantaneous $\mathrm{n}-\mathrm{V}$ characteristics at $x=3.0 \AA$ changes little with tip frequency. They are also similar to the static $n-V$ characteristics in Fig. 2(b). This is because at $\mathrm{x}=3 \AA$, tip junction resistance is small. The corresponding $\mathrm{RC}$ charging time is much smaller than the tip vibrating period, the instantaneous charging state on the nanoparticle is thus not affected by the tip vibration. As the tip moves away from the nanoparticle, $R_{T}$ increases 
rapidly leading to larger $R C$ charging time. Therefore charge imbalance on the nanoparticle can be affected at large tip-nanoparticle distance as the tip vibrating frequency increases, which is seen clearly from the instantaneous $\mathrm{n}-\mathrm{V}$ characteristics at the largest tip-nanoparticle distance $x=9.0 \AA$. The time-averaged $\mathrm{n}-\mathrm{V}$ characteristics approximates well the average between the instantaneous $\mathrm{n}-\mathrm{V}$ characteristics at $x=3.0 \AA$ and $9.0 \AA$.

Fig. 4 shows the number of electrons $n_{\text {shuttle }}$ being shuttled across the system per cycle of tip vibration, the displacement current and the electrostatic force on the tip averaged over one cycle of tip vibration at tip vibrating frequencies of $f=1,10,100 \mathrm{MHz}$ respectively. Note that $n_{\text {shuttle }}$ decreases with increasing tip frequency due to reduced efficiency of charge transfer from the central island to the tip. The capacitive part of the displacement current $I_{C a p}$ increases linearly with the tip-substrate bias voltage (solid line), but the "Coulomb Blockade" part of the displacement current $I_{C B}$ oscillates with the bias voltage, since $d n / d t$ changes sign during one cycle of the tip vibration. The peak and dip positions in $I_{C B}$ correlate closely with the step-wise change in the shuttled electrons with respect to the bias voltage. The magnitude of
$I_{C a p}$ increases linearly with increasing tip vibration frequency, but the increase in magnitude of $I_{C B}$ is less regular. We have also shown the time-averaged capacitive force $F_{C a p}$ and the discrete-electron force $F_{C B}$ on the tip separately in Fig. 4 Note that $F_{C a p}$ is quadratic voltagedependent and is attractive. In contrast, the discreteelectron force $F_{C B}$ is repulsive. The magnitude of $F_{C B}$ shows step-wise increase with bias voltage as more electrons are allowed to be shuttled, similar to the voltagedependence of $n_{\text {shuttle }}{ }^{15}$ The overall magnitude of $F_{C a p}$ and $F_{C B}$ is comparable. Unlike the displacement current, the magnitude of the discrete-electron force depends only weakly on the tip frequency through the frequency dependence of $n_{\text {shuttle }}$.

To conclude, we have presented a microscopic theory of mechanical modulation of single-electron tunneling through a molecular-assembled metal nanoparticle induced by a vibrating nanoprobe. The effects discussed here may be of interest for applications in nanomechanical chemical/bio-sensors.

This work was supported by the DARPA Moletronics program, the DoD-DURINT program and the NSF Nanotechnology Initiative.
* Author to whom correspondence should be addressed. Email: ayxue@chem.northwestern.edu

1 M.L. Roukes, Phys. World 14(2), 25 (2001); A. Cho, Science 299, 36 (2002).

2 A.N. Cleland and M.L. Roukes, Nature 392, 160 (1998); R.E.S. Polkinghorne and G.J. Milburn, Phys. Rev. A 64, 42318 (2001); A.D. Armour and M.P. Blencowe, Phys. Rev. B 64, 353111 (2001); A.D. Armour, M.P. Blencowe, and K.C. Schwab, Phys. Rev. Lett. 88, 148301 (2002); A.N. Cleland, Nature 424, 291 (2003); M.D. LaHaye, O. Buu, B. Camatota, and K.C. Schwab, Science 304, 74 (2004); A. D. Armour, M. P. Blencowe, and Y. Zhang, Phys. Rev. B 69, 125313 (2004).

3 L.Y. Gorelik, A. Isacsson, M.V. Voinova, B. Kasemo, R.I. Shekhter, and M. Jonson, Phys. Rev. Lett. 80, 4526 (1998).

4 C. Weiss and W. Zwerger, Europhys. Lett. 47, 97 (1999); N. Nishiguchi, Phys. Rev. B 65, 35403 (2001); T. Nord, L.Y. Gorelik, R.I. Shekhter, and M. Jonson, ibid. 65, 165312 (2002); A.D. Armour and A. Mackinnon, ibid. 66, 35333 (2002); T. Novotný, A. Donarini, and A.-P. Jauho, Phys. Rev. Lett. 90, 256801 (2003); D. Fedorets, L. Y. Gorelik, R. I. Shekhter, and M. Jonson, ibid. 92, 166801 (2004).

5 M.T. Tuominen, R.V. Krotkov, and M.L. Breuer, Phys. Rev. Lett. 83, 3025 (1999); A. Erbe, C. Weiss, W. Zwerger, and R.H. Blick, Phys. Rev. Lett. 87, 96106 (2001).

6 J.R. Petta, D.G. Salinas, and D.C. Ralph, Appl. Phys. Lett. 77, 4419 (2000).

7 Y. Xue and M.A. Ratner, Phys. Rev. B 68, 115407 (2003).

8 H. Park, J. Park, A.K.L. Lim, E.H. Anderson, A.P. Alivisatos, and P.L. McEuen, Nature 407, 57 (2000).

9 D. Dedorets, L.Y. Gorelik, R.I. Shehter, and M. Jonson, Europhys. Lett. 58, 99 (2002).

10 D. Boese and H. Schoeller, Europhys. Lett. 54, 668 (2001);
K.D. McCarthy, N. Prokof'ev, and M. T. Tuominen, Phys. Rev. B 67, 245415 (2003).

11 T. Novotný, A. Donarini, C. Flindt, and A.-P. Jauho, Phys. Rev. Lett. 92, 248302 (2004).

12 F. Pistolesi, Phys. Rev. B 69, 245409 (2004).

13 N.F. Schwabe, A.N. Cleland, M.C. Cross, and M.L. Roukes, Phys. Rev. B 52, 12911 (1995).

14 K. Nagano, A. Okuda, and Y. Majima, Appl. Phys. Lett. 81, 544 (2002); Y. Majima, K. Nagano, and A. Okuda, Jpn. J. Appl. Phys. 41, 5381 (2002).

15 Y. Suganuma, P.-E. Trudeau, and A.-A. Dhirani, Phys. Rev. B 66, 241405 (2002); Y. Suganuma, P.-E. Trudeau, A.-A. Dhirani, B. Leathem, and B. Shieh, J. Chem. Phys. 118, 9769 (2003).

16 F.J. Giessibl, Rev. Mod. Phys. 75, 949 (2003).

17 M. Buttiker, J. Low. Temp. Phys. 118, 519 (2000); B. Wang, J. Wang, and H. Guo, Phys. Rev. Lett. 82, 398 (1999).

18 C. Bruder and H. Schoeller, Phys. Rev. Lett. 72, 1076 (1994).

19 C. Schönenberger and S.F. Alvarado, Phys. Rev. Lett. 65, 3162 (1990); L.J. Klein and C.C. Williams, Appl. Phys. Lett. 79, 1828 (2001).

20 D.V. Averin and K.K. Likharev, in Mesocopic Phenomena in Solids, edited by B.L. Altshuler, P.A. Lee and R.A. Webb (Elsevier, Amsterdam, 1991).

21 Y. Xue and M.A. Ratner, Phys. Rev. B 68, 235410 (2003); Mater. Res. Soc. Symp. Proc. 735, C5.5 (2003).

${ }^{22}$ Y. Xue, S. Datta and M.A. Ratner, Chem. Phys. 281, 151 (2002); Y. Xue and M.A. Ratner, Phys. Rev. B 68, 115406 (2003); 69, 85403 (2004).

23 G.-L. Ingold and Yu.V. Nazarov, in Single Charge Tunneling, edited by H. Grabert and M.H. Devoret (Plenum, New York, 1992). 
${ }^{24}$ H. Schoeller and G. Schön, Phys. Rev. B 50, 18436 (1994); J. König, H. Schoeller and G. Schön, ibid. 58, 7882 (1998).

25 C. Liu and Q. Niu, Phys. Rep. 286, 349 (1997).

${ }^{26}$ Y. Meir and N.S. Wingreen, Phys. Rev. Lett. 68, 2512 (1992); A.P. Jauho, N.S. Wingreen and Y. Meir, Phys. Rev. B 50, 5528 (1994); H. Haug and A-P. Jauho, Quantum Kinetics in Transport and Optics of Semiconductors (Springer-Verlag, Berlin, 1996).

27 A.D. Becke, Phys. Rev. A. 38, 3098 (1988); M. Ernzerhof, J.P. Perdew and K. Burke, in Density Functional Theory I, edited by R. F. Nalewajski (Springer, Berlin, 1996).

28 Since the metal-molecule interaction is a local phenomenon, we neglect the curvature of the nanoparticle surface and calculate the electronic structure of metalmolecule-nanoparticle junction in the same way as that of the metal-molecule-metal junction. The transferred charge and therefore the background charge $n_{x}$ are obtained by integrating the (self-consistent) electron density distribution over the region occupied by the perturbed surface atoms of the nanoparticle.

29 R. Hoffmann, Rev. Mod. Phys. 60, 601 (1988); Y. Xue, S. Datta, S. Hong, R. Reifenberger, J.I. Henderson and C.P. Kubiak, Phys. Rev. B 59, 7852 (1999).

30 Y. Oyama, Y. Majima, and M. Iwamoto, J. Appl. Phys. 86, 7087 (1999); C. Wasshuber, Computational SingleElectronics (Springer-Verlag, Wien, 2001).

31 L.R.C. Fonseca, A.N. Korotkov, K.K. Likharev, and A.A. Odintsov, J. Appl. Phys. 78, 3238 (1995). 